\title{
Cognitive deficits in progressive supranuclear palsy, Parkinson's disease, and multiple system atrophy in tests sensitive to frontal lobe dysfunction
}

\author{
T W Robbins, M James, A M Owen, K W Lange, A J Lees, P N Leigh, C D Marsden, \\ N P Quinn, B A Summers
}

\begin{abstract}
Groups of patients with idiopathic Parkinson's disease, multiple system atrophy, and progressive supranuclear palsy or Steele-Richardson-Olszewski syndrome, matched for overall clinical disability, were compared using three computerised cognitive tests previously shown to be sensitive to frontal lobe dysfunction. On a test of planning based on the Tower of London task, all three groups were impaired, but in different ways. The groups with palsy and Parkinson's disease were slower in the measure of initial thinking time, whereas the group with multiple system atrophy was only slower in a measure of thinking time subsequent to the first move, resembling patients with frontal lobe damage. On a test of spatial working memory, each group showed deficits relative to their matched control groups, but the three groups differed in their strategy for dealing with this task. On a test of attentional set shifting, each group was again impaired, mainly at the extradimensional shifting stage, but the group with Steele-Richardson-Olszewski syndrome exhibited the greatest deficit. The results are compared with previous findings in patients with Alzheimer's disease or frontal lobe damage. It is concluded that these basal ganglia disorders share a distinctive pattern of cognitive deficits on tests of frontal lobe dysfunction, but there are differences in the exact nature of the impairments, in comparison not only with frontal lobe damage but also with one another.
\end{abstract}

(F Neurol Neurosurg Psychiatry 1994;57:79-88)

There is now overwhelming evidence that patients with basal ganglia disorders, including those with Parkinson's disease, Huntington's disease and progressive supranuclear palsy (or the Steele-RichardsonOlszewski syndrome, SRO), can exhibit characteristic cognitive deficits. ${ }^{1}$ However, the precise nature of these deficits, as well as their neural substrates, are still matters for debate. They appear to be different from those seen early in the course of Alzheimer's disease. ${ }^{2-5}$
For Parkinson's disease, some investigators have emphasised the 'frontal' or 'fronto-striatal' nature of the deficits, ${ }^{67}$ whereas others have described relatively focal neuropsychological abnormalities that occur, for example, in visuospatial function, ${ }^{8}$ and yet others have emphasised the similarities of the deficits to a syndrome of 'subcortical dementia'. 910

The original paper describing this syndrome was, in fact, illustrated with reports of several patients with SRO who exhibited forgetfulness, slowness of thought, changes in personality with apathy and depression, and an impaired ability to manipulate acquired knowledge. Others have also emphasised the frontal-lobe like nature of the deficits in SRO because of the failure of patients with SRO on classic tests of frontal lobe function such as the Wisconsin card sorting test and verbal fluency, as well as their tendency to exhibit frontal lobe 'signs', including enhanced grasp reflexes, motor impersistence, and utilisation behaviour. ${ }^{312} 13$ Indeed two of these studies ${ }^{12} 13$ showed that the two groups with Parkinson's disease and SRO matched for age and severity of intellectual deterioration, were impaired on tests of frontal lobe dysfunction, the group with SRO performing worse. From these studies of basal ganglia dysfunction, it is apparent that the relationship between the cognitive deficits seen in 'subcortical dementia' and frontal lobe dysfunction has yet to be resolved.

In contrast to the extensive investigation of cognitive dysfunction in these two conditions, there has been relatively little analysis of possible intellectual deficits in other informative progressive akinetic-rigid syndromes, which include multiple system atrophy. This disease is of particular interest for, in addition to the intrinsic striatal (caudate plus putamen) pathology, damage to the nigrostriatal dopamine pathway (particularly to the caudate nucleus) is at least equal, or even greater, to that seen in Parkinson's disease and SRO. ${ }^{14}$ Unsurprisingly, therefore, multiple system atrophy can initially prove difficult to differentiate clinically from idiopathic Parkinson's disease or SRO. ${ }^{15}$ Patients with atrophy generally have Parkinsonism as a major feature, attributed to striatonigral degeneration, but with the frequent additional presence of pyramidal, autonomic (Shy-Drager syndrome), or cerebellar (olivopontocerebellar atrophy) signs. ${ }^{15-17}$ 
The present study sought to compare groups of patients in tasks recently shown to be sensitive to frontal lobe dysfunction. These included a test of attentional set shifting ${ }^{18}{ }^{19}$ a test of planning based on the Tower of London task ${ }^{2021}$ and a test of spatial working memory. ${ }^{21}$ The importance of these tests is that, in certain cases, they have revealed significant differences in the precise pattern of deficits seen in various stages of Parkinson's disease and in patients with neurosurgical damage to the frontal lobes. ${ }^{22-24}$ This latter group of patients are significantly less accurate in their solutions to the Tower of London problems, but no slower than normal to initiate correct solutions. ${ }^{21}$ Patients with Parkinson's disease with relatively mild clinical disability, but sufficient to warrant treatment, in contrast are no less accurate than normal, but spend longer initially thinking about their solutions. ${ }^{22-24}$ Moreover, the patients with frontal lobe damage showed no evidence of employing the most advantageous strategy on the spatial working memory task, ${ }^{21}$ whereas the patients with Parkinson's disease, though showing equivalent overall levels of cognitive dysfunction, did exhibit evidence of using the strategy. ${ }^{22-24} \mathrm{~A}$ preliminary survey ${ }^{25}$ of the cognitive deficits in patients with multiple system atrophy has shown that they qualitatively resemble those of patients with frontal lobe damage rather than those with Parkinson's disease. These findings raise the possibility that the symptoms of frontal lobe dysfunction versus striatal damage may be differentiated using the same tests. Consequently, as there has been relatively little experimental detailed characterisation of slowness of thinking in SRO, nor of other frontal symptoms such as impaired set shifting and working memory, a detailed comparison with Parkinson's disease and multiple system atrophy in tests sensitive to frontal lobe dysfunction seemed to be warranted.

\section{Methods}

SUBJECTS

Patients with Parkinson's disease

The 24 patients included in this study were all outpatients at the Maudsley Hospital, London. In all cases, idiopathic Parkinson's disease was diagnosed by a consultant neurologist who also assessed the severity of clinical

Table 1 Patient characteristics

\begin{tabular}{lllclll}
\hline Group & $\begin{array}{l}\text { Stage } \\
\text { (mean) }\end{array}$ & $n$ & $M: F$ & Age & NART & $\begin{array}{l}\text { Duration } \\
\text { (years) }\end{array}$ \\
\hline SRO & 3.8 & 18 & $9: 9$ & $64 \cdot 1(1 \cdot 35)$ & $108 \cdot 7(2 \cdot 15)$ & $4 \cdot 7(0 \cdot 8)$ \\
Controls & & 18 & $7: 11$ & $64 \cdot 2(1 \cdot 66)$ & $109 \cdot 2(1 \cdot 6)$ & \\
MSA & 3.7 & 16 & $14: 2$ & $51 \cdot 1(1 \cdot 99)$ & $111 \cdot 5(2 \cdot 25)$ & $6 \cdot 2(0 \cdot 7)$ \\
Controls & & 16 & $8: 8$ & $54 \cdot 6(1 \cdot 88)$ & $112 \cdot 6(1 \cdot 73)$ & \\
PD & 3.5 & 24 & $16: 8$ & $63 \cdot 2(2 \cdot 46)$ & $107 \cdot 9(2.56)$ & $10 \cdot 2(1 \cdot 57)$ \\
Controls & & 24 & $7: 17$ & $61 \cdot 5(1 \cdot 28)$ & $111 \cdot 6(1 \cdot 34)$ & \\
\hline
\end{tabular}

Standard errors are shown in parentheses. Stage $=$ Hoehn \& Yahr Stage ${ }^{26} ;$ NART $=$ Nationa Adult Reading $\mathrm{Test}^{30} ;$ SRO $=$ Steele-Richardson-Olszewski syndrome; MSA $=$ Multiple System Atrophy; PD = Parkinson's disease. symptoms according to the Hoehn and Yahr rating scale ${ }^{26}$ while the patients were on treatment. All patients were receiving levodopa preparations, to which they had responded, either alone or in combination with other medication.

Exclusion criteria for these patients included clinical dementia assessed using both the Mini-mental state examination (MMSE) ${ }^{27}$ and the Kendrick object learning test (KOLT). ${ }^{28}$

\section{Patients with Steele-Richardson-Olszewski Syndrome}

The 18 patients with SRO were diagnosed at the National Hospital for Neurology and Neurosurgery and at the Maudsley Hospital, London, according to the criteria set out by Lees. ${ }^{29}$ The duration of disease averaged 4.7 years taken from the initial symptoms. Six patients were on dopaminergic medication at the time of testing and one patient was receiving anticholinergic medication.

\section{Patients with multiple system atrophy}

All 16 patients were diagnosed at the National Hospital, according to criteria set out by Quinn. ${ }^{15}$ The duration of disease averaged six years taken from the time of initial symptoms. Only two of the 16 patients were not receiving any medication. All the remaining patients were receiving levodopa or bromocriptine preparations. Two patients were also receiving the anticholinergic drug benzhexol.

\section{Control subjects}

Three groups of healthy control subjects $(n=52)$ were chosen to match the groups of patients, as closely as possible with respect to age and premorbid verbal IQ as assessed using the National adult reading test (NART). ${ }^{30}$ These subjects were drawn from a large pool of control volunteers at the North East Age Research panel in Newcastle Upon Tyne, United Kingdom.

In the attentional set shifting task, the three patient groups were compared with a large group of normal control volunteers $(\mathbf{n}=$ 79 , mean age $=58.8 \quad(\mathrm{SE}=0.9)$, mean NART IQ $116.0(0.9)$ between the ages of 41 and 69 drawn from the North East Age Research panel in Newcastle Upon Tyne and from the Newcastle, Cambridgeshire and London areas. Informed consent was obtained for all patients and normal volunteers.

Table 1 shows a summary of characteristics for the three patient groups and their controls. One way analysis of variance confirmed that the patient groups were all well matched with their respective control groups in terms of age and NART IQ estimate. In terms of the Hoehn and Yahr scale of clinical disability, all patients received ratings of between III and $\mathrm{V}$. The distribution of these ratings (III IV and V) was: multiple system atrophy, 85 3; Parkinson's disease 1491 ; SOR 792 . Contingency table analysis of these scores (see over) showed that there were no signifi- 
cant differences between the three groups in the distribution of these scores $(2 \mathrm{i}=4.65, \mathrm{df}$ $=4$; not significant).

\section{MATERIALS AND PROCEDURES}

The main testing procedures were taken from the Cambridge Neuropsychological Test Automated Battery (CANTAB), a series of computerised paradigms run on an Acorn BBC Master microcomputer with a high resolution Microvitec colour monitor and a Microvitec (Touchtech 501) touch sensitive screen. The general rationale and use of the battery for clinical investigations has recently been explained in some detail. ${ }^{23}$ Subjects were seated approximately $0.5 \mathrm{~m}$ from the monitor and it was explained that they would have to respond to stimuli by touching the screen.

\section{Spatial short term memory task}

In this computerised Corsi block tapping task, ${ }^{31}$ spatial short term memory capacity was determined from the ability of subjects to remember a sequence of squares on the screen, as described in detail in an earlier publication. ${ }^{21}$ Spatial short term memory span was calculated as the highest level at which the subject successfully recalled at least one sequence of boxes.

\section{Spatial working memory task}

In this task the subject was required to search through spatial arrays of boxes to find tokens. Importantly, once a blue token had been found within a particular box, then that box would never be used to hide another token. On each trial, the total number of blue tokens to be found corresponded to the number of boxes on the screen, as every box was used just once.

Errors were scored according to the number of occasions on which a subject returned to open a box in which a blue counter had already been found. After four practice trials with three boxes, there were four test trials with each of four, six, and finally eight, boxes.

In the present study, the three patient groups were compared in terms of the total number of errors summed across the 12 test trials. One possible strategy for completing this task is to follow a predetermined search sequence, beginning with a particular box and then returning to start each new sequence with that same box as soon as a token has been found. A previous investigation has shown that impaired performance on this task may be related to inefficient use of this particular search strategy in neurosurgical patients with frontal lobe excisions. ${ }^{21}$ The extent to which each group used this repetitive searching pattern as a strategy for approaching the problem was estimated from the number of search sequences starting with the same box, within each of the more difficult six and eight box problems. The total of these scores provided a single measure of strategy for each subject, with a high score (many sequences beginning with a different box) representing low use of the strategy and a low score (many sequences starting with the same box) representing more extensive usage.

\section{Planning task}

This is a modification of the Tower of London $\operatorname{task}^{20}$ in which the subject has to move coloured 'balls' on the screen from an initial arrangement to one corresponding to the goal arrangement shown in the top half of the screen, as described in detail previously. ${ }^{21} 25$

The starting position of the balls was varied such that in any particular trial the solution could only be reached after a minimum of two, three, four or five moves. Subjects were instructed to examine the position of the balls at the beginning of each problem and attempt to solve it in the minimum possible number of moves. This was both given to them verbally and displayed on the screen throughout each trial. They were encouraged not to make the first move until they were confident that they could execute the entire sequence needed to solve the problem. The maximum moves allowed corresponded to twice the minimum number possible plus one, or plus two in the case of 'five move' problems.

For each test problem, a 'yoked control' condition was employed to provide baseline measures of motor initiation and execution times. On each trial of this control condition, the subject was required to follow a sequence of single moves executed by the computer in the top half of the screen by moving the corresponding ball in the lower arrangement. The measurement of selection and execution latencies in this control condition provided baseline estimates of motor initiation and execution times ( $\operatorname{se}^{21}$ for further details).

\section{Attention set shifting}

The computerised attentional set shifting paradigm has been described in detail elsewhere. ${ }^{1819}$ Briefly, subjects are trained on a series of visual discriminations which vary in two perceptual dimensions, one of which is correct or relevant and one of which is incorrect or irrelevant, using feedback provided automatically by the computer. At critical points subjects are required first to maintain attention to different examples within the same dimension (intradimensional shift) and then to shift attention to the previously irrelevant dimension (extradimensional shift). The other stages are defined and explained in previous papers and in the Results section. For each stage, continuation to the next one was dependent on a criterion of six successive correct responses being reached. If criterion was not reached by the 50th trial of a stage, then the test was discontinued and subjects did not proceed to the following stage. More detailed explanation and rationales for the exact design of the test can be found in previously published articles. ${ }^{18} 1932-34$

\section{STATISTICAL ANALYSIS}

For most of the dependent variables, analysis of variance was used. Data were transformed 
where appropriate - that is, when there was a positive skew on latency variables. For most of the test variables, the analysis of variance model was a two factor design that included a between subjects factor (group) and a within subject factor - for example, difficulty level. ${ }^{35}$ As the group with multiple system atrophy were younger and showed a tendency towards a higher IQ than the other two patient groups, the patient groups were each compared with an appropriate age and IQ matched control group.

For the attentional set shifting task, the data for the numbers of subjects passing or failing each stage of the test were cast into contingency tables and analysed using the likelihood ratio method. ${ }^{3637}$ This method is particularly useful, firstly, for analysing data with small cell frequencies, as occurs in some of the data to be presented, and secondly, for partitioning inhomogeneities in the contingency table by additive, orthogonal contrasts. ${ }^{37}$ The resulting 'information' statistic (2i) is distributed as $\chi^{2} \cdot{ }^{36}$

\section{Results}

SPATIAL SHORT TERM MEMORY TASK

The three patient groups were independently compared with their respective control groups in terms of the number of squares that were touched in the correct serial order. Mean values and corresponding standard errors for the six groups are shown in fig 1 . One way analyses of variance showed that the groups with Parkinson's disease and SRO were significantly impaired on this measure $(F(1,46)=$ 15.86, $\mathrm{p}<0.001$ and $\mathrm{F}(1,34)=7.65$, $\mathrm{p}<0.01$ respectively). In contrast, the patients with multiple system atrophy were not significantly impaired $(F(1,30)=2.9)$ compared with their matched control group.

SPATIAL WORKING MEMORY TASK

The mean total numbers of 'between search errors' made by the three patient groups and

Figure 1 Performance on the spatial span task for the groups with multiple system atrophy (MSA), system atrophy (MSA),
Parkinson's disease (PD), Steele-RichardsonOlszewski syndrome (SRO) and their matched controls. Values shown are mean maximum spans $(S E)$.



their controls are presented in fig 2. There was a highly significant difference between the group with Parkinson's disease and their controls $(F(1,46)=7 \cdot 66, p<0 \cdot 01)$, between the group with SRO and their controls $(\mathrm{F}(1,34)=7.49, \mathrm{p}<0.01)$ and between the group with multiple system atrophy and their controls $(F(1,35)=5.45, p<0.05)$. Within search errors were at a low level and not significantly increased in any of the three groups.

The measure of strategy employed in this task was scored on a scale of $1-37$, with lower scores representing more efficient use of the strategy. The best possible score of 1 was obtained when, within each of the more difficult six and eight box problems, the same box was used to initiate each search sequence. Conversely, if every search within each of the problems was started with a different box, the maximum score of 37 was obtained. The mean (SE) scores were $17.5(0.74)$ for patients with Parkinson's disease, $20.05(0.84)$ for patients with SRO, and 16.6 $(0.39)$ for those with multiple system atrophy. Their corresponding control group means (SE) were $15.1(0.96), \quad 16.4(1.36)$ and $14 \cdot 8(0 \cdot 20)$. Only the group with SRO differed significantly from their controls in terms of this strategy measure $(F(1,35)=4 \cdot 3$, $\mathrm{p}<0.05)$, although in the Parkinsonian group the difference approached significance $(\mathrm{F}(1,46)=3 \cdot 96, \mathrm{p}=0.052)$.

The relationship between the strategy score and performance on the spatial working memory task was further examined using Pearson's product moment correlation coefficient, r. In the total control group, there was a highly significant correlation between the total number of between search errors and the extent to which the strategy described above was used $(\mathrm{r}=0.74, \mathrm{p}<0.001)$. Similarly, in patients with SRO, a positive

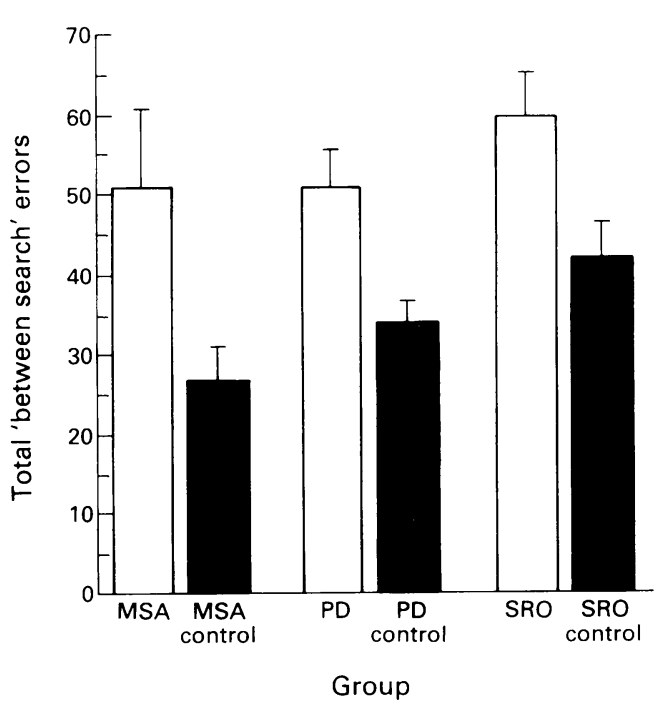

Figure 2 Between search errors on the spatial working memory task for the groups with multiple system atrophy (MSA), Parkinson's disease (PD), and

Steele-Richardson-Olszewski syndrome (SRO) and their matched controls. Values shown at each level of difficulty are mean numbers of between search errors (SE). 
correlation between these variables was found (SRO, $\mathrm{r}=0.56, \mathrm{p}<0.01$ ). However, in the other two patient groups, there were no such significant correlations between strategy and task performance, $r=0.46$, and 0.30 , $\mathrm{p}>0.05$, respectively).

These results show that all three patient groups made significantly more 'between search errors' than their controls on this test of spatial working memory. In all three cases this impairment may be related, in varying degrees, to inefficient use of a particular repetitive searching strategy. Although the index of strategy was only significantly reduced in the group with SRO, the subsidiary correlational analysis of its relationship to task performance showed clear abnormalities in the other two groups.

\section{PLANNING TASK}

Across the 12 test problems, two measures relating to the number of moves required to reach solution were calculated. The 'mean

Figure 3 Mean (SE) initial thinking times in seconds on the Tower of London tasks at various levels of difficulty for the groups with multiple system atrophy (MSA), Parkinson's disease (PD) and Steele-RichardsonOlszewski syndrome (SRO) and their matched controls. Difficulty refers to $2,3,4$, and 5 move problems.
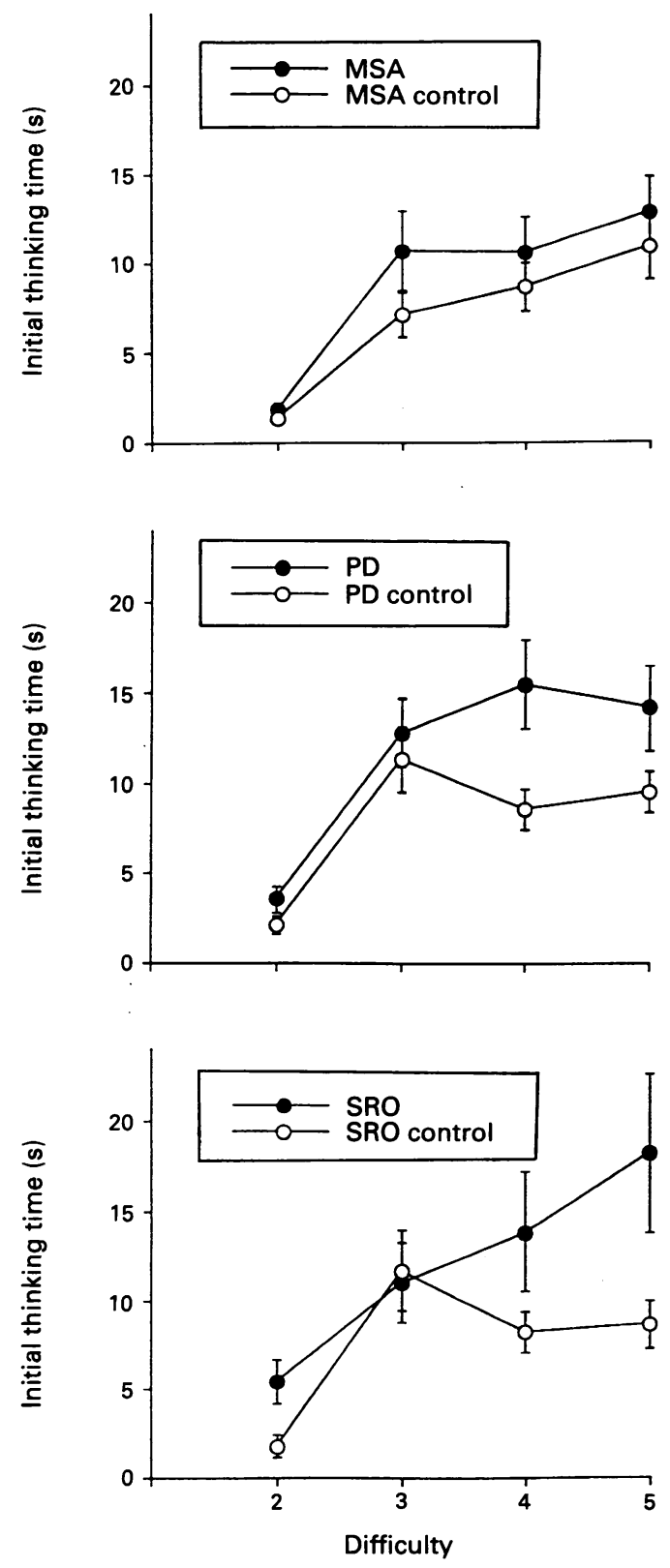

number of moves above the minimum possible' provided a general measure of group performance at each level of difficulty. The 'proportion of problems solved in the minimum number of moves' provided more specific information about task difficulty and its effect on the patient and control groups.

There were no significant differences between any of the three patient groups and their respective controls in terms of the number of moves to solution. In terms of the number of problems solved in the minimum number of moves, only the group with SRO differed significantly from their control group $(F(1,34)=4 \cdot 13, p<0.05)$. In all groups, there were significant main effects of task difficulty, although there were no significant interactions between the task difficulty and group factors.

Baseline measures of motor initiation time and motor execution time were extracted from the 12 "yoked control" trials. The motor initiation time represented the mean time between the onset of each problem and the completion of the first selection-that is, a correct touch of the required ball. For the patient groups, average initial movement times varied between 2.81 and $3.72 \mathrm{~s}$ for patients with Parkinson's disease, 5.09 and 6.62 for patients with SRO, and 2.98 and $4.42 \mathrm{~s}$ for patients with multiple system atrophy. Their corresponding control group ranges were $2 \cdot 35-4 \cdot 13,2 \cdot 70-3 \cdot 89$, and $2 \cdot 06-2 \cdot 45 \mathrm{~s}$. A similar pattern was found for the subsequent movement time data.

The groups with SRO and multiple system atrophy were significantly slower than their controls both in terms of initial and subsequent movement time. The group with Parkinson's disease were only significantly impaired in terms of subsequent movement time $(F(1,31)=4.66, p<0.05)$.

The movement times discussed above were used to derive estimates of planning or thinking time in the main task. Two main estimates were calculated.

The initial thinking time was the interval between the presentation of the problem and the first touch of a ball, minus the corresponding motor initiation time. The subsequent thinking time was the time between the first touch of a ball and the completion of the entire problem minus the total motor execution time derived from the corresponding control problem. Because subsequent thinking time varied with the length of the problem, this measure was divided by the number of moves actually made when tackling that same problem to give an estimate of the thinking time per move. The initial and subsequent thinking times for the three patient groups and their controls are shown in figs 3 and 4.

The patients with Parkinson's disease and SRO were significantly slower than their controls in terms of the initial thinking time measure $(F(1,42)=5.03, p<0.05$ and $(F(1,31)$ $=5.07, p<0.05$, respectively). In both cases, there were significant main effects of task difficulty although there were no significant 
Figure 4 Mean (SE) subsequent thinking times in seconds on the Tower of London tasks at various levels of difficulty for the groups with multiple system atrophy (MSA), Parkinson's disease (PD) and Steele-RichardsonOlszewski syndrome (SRO) and their matched controls. Difficulty refers to $2,3,4$, and 5 move problems.
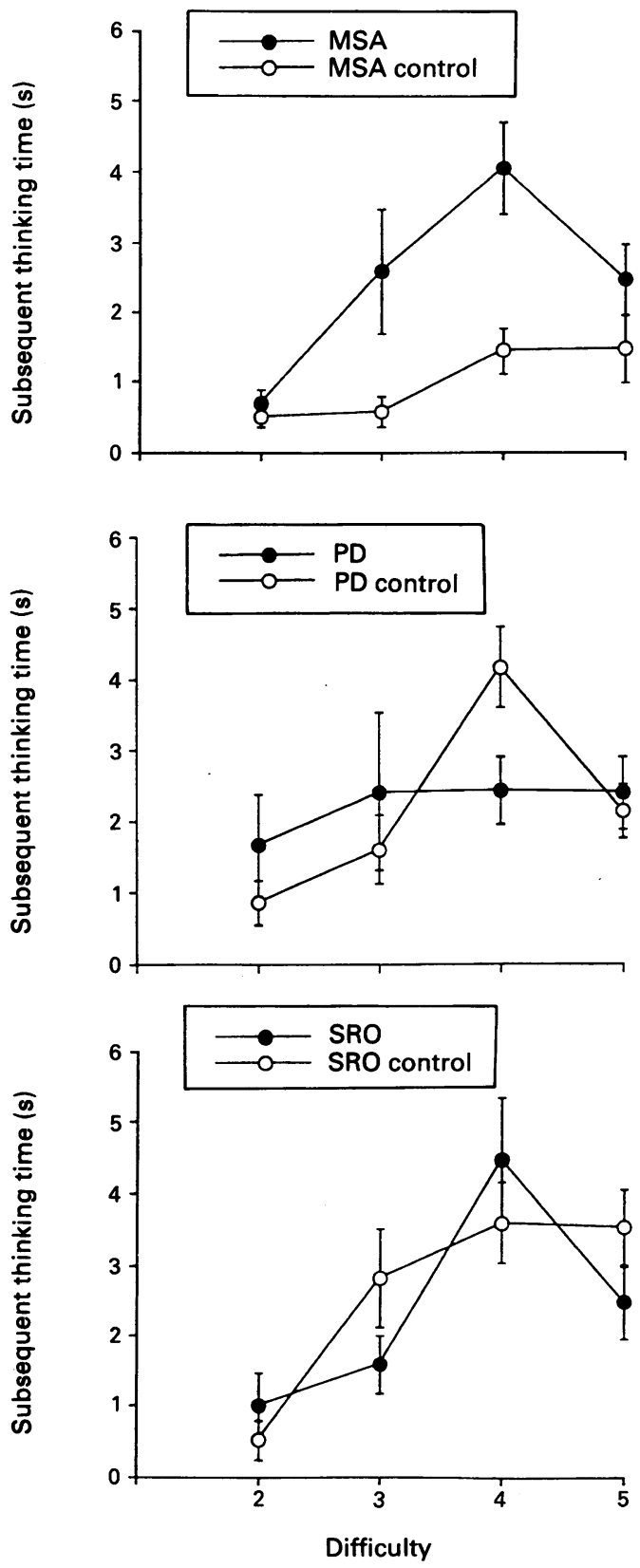

interactions between the task difficulty and group factors. In contrast, only the group with multiple system atrophy were significantly slower than their controls in terms of the subsequent thinking time measure $(F(1,30)=11 \cdot 24, p<0.005)$. There were significant interactions between task difficulty and group in each of the patient groups $(\mathrm{F}(3,90)=3.14, \mathrm{p}<0.05$ for multiple system atrophy; $(F(3,123)=2.95, p<0.05$ for Parkinson's disease; and F $(3,90)=2 \cdot 81, \mathrm{p}<$ 0.05 for SRO).

These results confirm that, although all three patient groups had difficulty on this test of planning ability, their impairments were both quantitatively and qualitatively different. Thus the patients with multiple system atrophy show significantly retarded subsequent thinking time in the absence of deficits in initial thinking time or thinking accuracy, whereas the Parkinson's patients show the exact converse pattern of impairments. By comparison with these two groups, the group with SRO more closely resembles the group with Parkinson's disease, although the effects on thinking accuracy did not quite reach significance for the latter.

\section{ATTENTIONAL SET SHIFTING TEST}

The incidences of subjects successfully completing each stage of the discrimination learning test are shown separately for each group in fig 5 . As can be seen, $82 \%$ of the control group successfully completed the whole test, whereas the three patient groups had significantly lower success rates $(55 \%$ for Parkinson's disease; $56 \%$ for multiple system atrophy; and $24 \%$ for SRO). Analysis of the contingency table of raw scores using the likelihood ratio method revealed a highly significant difference among the groups $(2 \mathrm{i}=$ $27 \cdot 70, \mathrm{df}=3, \mathrm{p}<0 \cdot 001)$. Further analysis using orthogonal contrasts showed that the three patient groups were significantly different from the controls $(2 \mathrm{i}=20.91, \mathrm{df}=1)$. The three patient groups were significantly different among themselves $(2 \mathrm{i}=7 \cdot 61, \mathrm{df}=$ $2, \mathrm{p}<0.05$ ), a result accounted for largely by the inferior performance of the group with SRO relative to the other two $(2 \mathrm{i}=6 \cdot 75, \mathrm{df}=$ $1, \mathrm{p}<0.01)$. When the incidence data were analysed at each stage, taking into account the number of subjects actually attempting that stage, a qualitatively similar pattern of results was obtained. Thus, for example, of those subjects attempting the extradimenstonal shift, there was again a significant over-

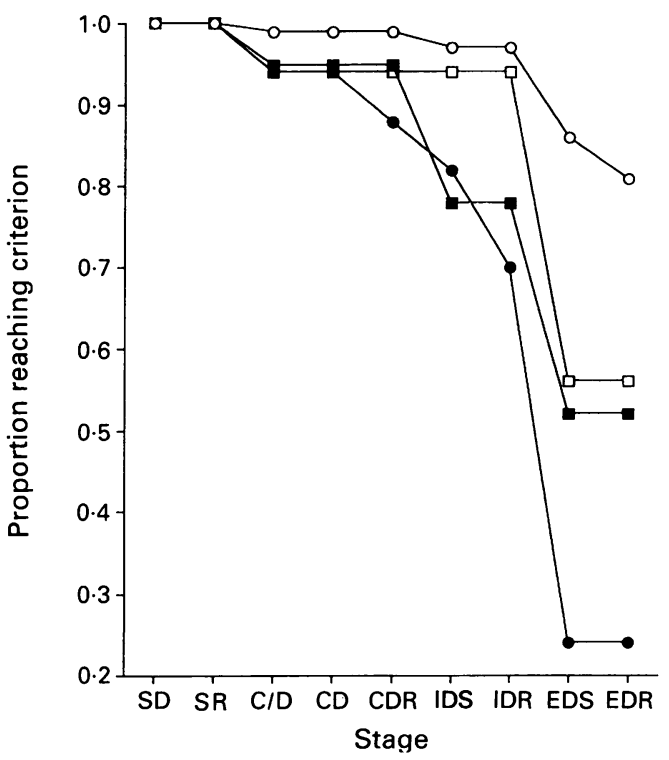

Figure 5 Proportion of subjects successfully passing each stage of the attention set shifting paradigm. Key: ( $\square$ ) multiple system atrophy; (1) Parkinson's disease; (O) Steele-Richardson-Olszewski syndrome; (O) controls. SD = simple discrimination; $S R=$ simple reversal; $C / D=$ compound discrimination with separated elements; $C D=$ compound discrimination with superimposed elements; $C D R=$ compound discrimination reversal; $I D S=$ intradimensional shift; IDR = intra-dimensional shift reversal; $E D S=$ extra-dimensional shift; EDR = extra-dimensional shift reversal. Note the large attrition in the patient groups at the extra-dimensional shift. 
all group effect $(2 \mathrm{i}=21.59, \mathrm{df}=3, \mathrm{p}<$ 0.001 ), and the controls were significantly different from the combined patient groups $(2 \mathrm{i}=17 \cdot 15, \mathrm{df}=1, \mathrm{p}<0.001)$. Although there was a clear tendency for the group with SRO to perform worse (\% success rates $70 \%$ for Parkinson's diseases; $60 \%$ for multiple system atrophy; and $33 \%$ for SRO) this did not reach significance $(2 \mathrm{i}=4 \cdot 30, \mathrm{df}=2$, not significant).

\section{Discussion}

This study has compared groups of patients with different forms of basal ganglia disorder, with control groups appropriately matched for age and premorbid IQ, on three tests that have been shown to be sensitive to frontal lobe dysfunction. The three groups were matched for general clinical disability, as measured by the Hoehn and Yahr rating. The results indicate that all three groups were impaired on the tests of frontal lobe function, although the precise pattern of deficits generally differed from those previously described following neurosurgical damage to the frontal lobes $^{1921}$ (table 2). There were minor qualitative differences among the three forms of basal ganglia disorder.

The frontal tests employed included a computerised version of the Tower of London planning task, adapted to measure not only the efficiency of problem solving, but also the speed of thinking, as reflected by the latency to initiate solution attempts after correction for motor slowing using a yoked control task. Using similar methodology, it was also possible to measure the time taken to think about the problems during the sequence of moves. These measures have previously distinguished patients with Parkinson's disease from those with frontal lobe damage. ${ }^{21-24}$ Parkinsonian patients on medication with relatively mild clinical disability-that is, Hoehn and Yahr ratings of 1 and 2-have been shown to be slower on the initial thinking time measure, but to be no less accurate than control subjects at solving the problems, ${ }^{21-24}$ whereas frontal lobe patients exhibited no initial slowing of thinking, but showed impaired efficiency of problem solution and lengthened subsequent thinking times. ${ }^{21}$ In addition to confirming the lengthened initial thinking time of Parkinsonian patients on medication, this study, together with other results, ${ }^{23}{ }^{24}$ demon- strates that those patients with more severe clinical disability are also impaired in the accuracy of solutions, as indexed by the minimum moves measure, and to a minor degree, in subsequent thinking time.

It is possible that the lengthened initial thinking times in Parkinson's disease reflects the clinical concept of bradyphrenia, this having been discussed in some detail elsewhere ${ }^{22} 23$ and so it was of particular interest to compare the disease with SRO directly, as slowing of thought processes has been considered to be a prominent feature of the second condition. Our results indicate that, indeed slowness of thinking is found in the group with SRO; in fact their pattern of performance on the Tower of London test of lengthened initial thinking time, largely unchanged subsequent thinking time and impaired accuracy of problem solving, is similar to that of the Parkinsonian group matched for overall clinical disability, the pattern in both groups contrasting with that seen in patients with frontal lobe damage.

Although the patients with SRO exhibited lengthened initial thinking times, it was perhaps surprising that their deficit was no greater than in patients with Parkinson's disease, where bradyphrenia is a less prominent clinical feature. This perhaps indicates that bradyphrenia might represent a specific form of cognitive slowing. It is of interest to note that the patients with SRO in this study had greatly lengthened latencies on the yoked control task in comparison to the other two patient groups, even despite their similar degree of overall clinical disability. This task itself had quite complex requirements which effectively controlled for overall sensorimotor slowing in the group with SRO. Nevertheless, it is apparent that when the time spent actually planning the solutions to the problems is corrected for these sensorimotor disabilities, there is no obvious quantitative difference in thinking time between the groups with Parkinson's disease and SRO.

In general terms these neuropsychological results are in agreement with those of other investigators ${ }^{1338}$ although there are some differences of detail and interpretation. For example, Dubois et $a l,{ }^{13}$ in a comparative study of 10 patients with SRO and 33 with Parkinson's disease, used a similar type of subtractive methodology to that employed by us in the Tower of London task, but applied instead to a choice reaction time paradigm.

Table 2 Summary of results and other studies

\begin{tabular}{|c|c|c|c|c|c|c|}
\hline & $\begin{array}{l}\text { Unmedicated } \\
P D^{\star}\end{array}$ & $\begin{array}{l}\text { Medicated } \\
P D\end{array}$ & $M S A$ & $S R O$ & Frontal† & $\begin{array}{l}\text { Alzheimer type } \\
\text { dementia }\end{array}$ \\
\hline Span & $\checkmark$ & $x$ & $\checkmark$ & $x$ & $\checkmark$ & $\times$ \\
\hline Spatial working memory ('between search' errors) & $\times$ & $x$ & $x$ & $x$ & $x$ & $x \neq$ \\
\hline Minimum move solutions (Tower of London) & $\checkmark$ & $\checkmark$ & $\checkmark$ & $\times$ & $\times$ & $\times \coprod^{\top}$ \\
\hline Initial thinking time (Tower of London) & $\checkmark$ & $x$ & $\checkmark$ & $\times$ & $\checkmark$ & $\times \mathbf{5}$ \\
\hline Subsequent thinking time (Tower of London) & $x$ & $\checkmark$ & $x$ & $\checkmark$ & $x$ & $\times \mathbb{5}$ \\
\hline Attentional set shifting & $x$ & $x$ & $x$ & $\times$ & $\times \|$ & $\sqrt{\operatorname{ql} x} \times \star \star$ \\
\hline
\end{tabular}


This study showed that the patients with SRO spent relatively longer than controls in a measure of analysis time, which reflected the additional central processing latency of more complex reaction time contingencies for the same motor response. No such effect was found, in the patients with Parkinson's disease, however, although these had equivalent Hoehn and Yahr disability ratings. It may well be the case, as Dubois et al suggest, that reaction time procedures are too simple for patients with Parkinson's disease who usually exhibit cognitive dysfunction only for complex situations. Such procedures may be more optimal for assessing bradyphrenia in SRO, a possibility consistent with our present observations of the poor performance of this group on the yoked control task.

In related work, Blin et al ${ }^{39}$ make a good case that in SRO the fontal lobe like cognitive deficits are in fact related to frontal metabolic activity, rather than activity in the caudate nucleus, as measured using positron emission tomography. Unlike earlier studies with smaller numbers of patients ${ }^{4041}$ they found a small but significant correlation between the compound frontal score and an index of frontal lobe activity, the frontal-occipital cortex metabolic ratio. Although a relationship between frontal lobe activity and performance on our cognitive tests would not be surprising, it is apparent that the pattern of deficit on the Tower of London task for the group with SRO is not exactly the same as shown for patients with frontal lobe damage. On the other hand, our finding of impaired planning accuracy in the group with SRO is not in agreement with the view that 'problem solving behaviours appear[ed] relatively intact in patients with SRO' ( ${ }^{38}$, p. 557).

Although patients with Parkinson's disease and SRO showed contrasting impairments to those with frontal lobe lesions on the Tower of London test, the patients with multiple system atrophy, a condition involving diffuse subcortical damage that includes the basal ganglia, exhibited a comparable pattern, in particular showing sparing of initial thinking time, but slowing of subsequent thinking time. There were, however, several difficulties involved in making comparisons between the patients with multiple system atrophy and the other basal ganglia disorders, even though the three groups were grossly matched for clinical disability. In particular, these patients were younger and were slightly more intelligent, thus necessitating the use of separate IQ and age-matched control groups. This resulted in several problems, as spatial span, spatial working memory and certain latency measures on the Tower of London task exhibited obvious age-related changes. Thus, for example, subsequent thinking time was impaired in this group only relative to the performance of their control group, rather than in absolute terms. The pattern of change in the latency scores for initial thinking time (where there was no difference whatsoever from the control group) and subsequent thinking time, was clearly distinct from that of the other two patient groups. In addition, although the spatial span of this group was comparable to that of the control groups for SRO and Parkinsonism, they were nevertheless worse than their own control group, although this trend did not reach significance.

The impairments in planning function found in the three patient groups were also paralleled by substantial deficits in the spatial working memory task, as shown in particular by the greatly increased numbers of between search errors, which were comparable in magnitude to those reported previously for patients with frontal lobe damage. ${ }^{21}$ Within search errors, however, which are elevated in patients with frontal lobe damage, were at a low level in each of the groups.

The spatial working memory task requires a self-ordered, well-organised search to maintain high levels of performance, which presumably depends upon executive functions such as the implementation of a searching strategy. The deficits in performance on this task in patients with frontal lobe damage have indeed been related directly to such a strategic impairment. ${ }^{21}$ This contrasts with the performance of patients with probable Alzheimer's disease who show no impairments in the use of the same strategy, while exhibiting massive deficits in spatial working memory performance. ${ }^{42}$ Thus, it is apparent that the contribution of both executive and mnemonic factors to efficient performance can be differentiated on this task. In terms of the present study, the patients with basal ganglia disorders showed both types of deficit, in varying degrees.

For example, the groups with SRO and Parkinson's disease showed some impairment in a measure of the efficient use of a well defined strategy for mediating the spatial working memory task, similar to patients with frontal lobe damage. ${ }^{21}$ The normal positive relationship between the use of this strategy and spatial working memory performance was absent in multiple system atrophy, however, as well as in the Parkinsonian group. These findings suggest that the spatial working memory deficits in the second two groups cannot simply be explained in terms of executive dysfunction, and reflect additional deficiencies of spatial memory capacity.

The discrimination learning and set shifting paradigm was also sensitive in detecting deficits in each of the three groups, although performance in the group with SRO was especially weak, with only about $25 \%$ of the patients successfully completing the entire test. This deficit is especially significant, because it cannot be attributed to the disturbed eye movements in this disorder, as the stimulus dimensions for the discrimination of the compound stimuli were superimposed and the time factor was not relevant. Many of the failures on this test were due to the special difficulty shown by the patients at the extradimensional shift stage, when attentional set has to be shifted to the formerly irrelevant stimulus dimension. The deficit in Parkinsonism has been reported previously, ${ }^{18}$ 
and it is important to note that it is present even in the earliest stages of the disease, before the onset of medication, being the most sensitive of our frontal lobe tests to early in the course Parkinson's disease. ${ }^{23}$ Moreover, the deficit appears to be ameliorated by levodopa. ${ }^{1834}$ Firstly, the performance of unmedicated, early in the course patients with Parkinson's disease is, if anything, inferior to that of patients on medication who are further in the course of the disease..$^{18}$ Secondly, withdrawal of levodopa from severely affected patients produced a selective and large deficit in performance. ${ }^{34}$ Therefore, there is clearly a dopaminergic component to performance on this task.

These results are relevant to two aspects of the present study. All of the Parkinson's patients, most of the patients with multiple system atrophy, but less than half of the patients with SRO were receiving levodopa (or bromocriptine) medication. Thus, the degree of deficit in the first two groups may have been masked to some extent. Therefore, although the deficits in this paradigm were greater in the group with SRO it is possible that they would have been less evident in comparison with unmedicated patients from the other two groups. For the group with SRO, $2 / 6$ of the patients receiving dopaminergic medication were successful, whereas this was true for a smaller proportion $(2 / 11)$ of the remaining patients not receiving medication, again consistent with some dopaminergic benefit to performance on this task.

The attentional set shifting test is also of some theoretical importance because, like the Wisconsin card sorting test, ${ }^{3}$ it is relatively insensitive to deficits early in the course of Alzheimer's disease. ${ }^{33}$ Therefore, it appears that this capacity is selectively impaired in patients with basal ganglia and frontal lobe dysfunction, and might be dependent on the integrity of functional neuronal loops connecting the basal ganglia with the frontal cortex. ${ }^{43}$

It should also be emphasized that the deficits shown by our patient groups cannot easily be attributed to global intellectual deterioration. On routine clinical assessment, few of the patients with multiple system atrophy and none of the cases with Parkinson's disease showed evidence of any degree of dementia. Moreover, there was little consistent evidence of visual memory dysfunction in the group with multiple system atrophy. ${ }^{25} \mathrm{On}$ the other hand, Parkinsonian patients, with severe disability, do show significant decrements in most of the tests in the CANTAB visual memory battery. ${ }^{54}$ Therefore, it is apparent that the cognitive deficits in Parkinson's disease are broader in nature than those in multiple system atrophy, including impairments associated with cortical regions other than the frontal lobe.

In the case of the group with SRO, approximately half the patients showed a NARTWAIS-R discrepancy of 10 or more points and could be classified as having a significant degree of generalized cognitive deterioration.
However, post hoc comparisons between the two resultant subgroups failed to show any significant differences in performance on the three tests of frontal lobe function, with the exception of minimum move solutions. Therefore, it appears likely that more generalized dementia in the group with SRO is associated with a different neural substrate than the specific impairments of frontostriatal function described here.

In conclusion, all three of these basal ganglia disorders show significant cognitive impairments on tests sensitive to frontal lobe dysfunction. Overall, the gross similarity in the results of these computerised tests contrasts with the obvious clinical differences associated with these diseases, but may indicate a common and fundamental syndrome of cognitive dysfunction, a frontostriatal dementia. This is not a generalised dementia because it contrasts markedly with that seen in early Alzheimer's disease. Moreover, we have shown that there are some qualitative differences between the patterns of deficit in these basal ganglia diseases which may indicate subtle differences in underlying frontostriatal pathology.

This work was supported by a Programme grant from the Wellcome Trust.

1 Brown RG, Marsden CD. Subcortical dementia: The neuropsychological evidence. Neuroscience 1988;25: 363-87.

2 Moss M, et al. Differential patterns of memory loss among patients with Alzheimer's disease. Huntington's disease and alcoholic Korsakoff syndrome. Arch Neurol 1986;43: 239-46.

3 Pillon B, Dubois B, L'Hermitte F, Agid Y. Heterogeneity of cognitive impairment in progressive supranuclear palsy, Parkinson's disease and Alzheimer's disease. palsy, Parkinson's disease

4 Sagar HJ, Cohen NJ, Sullivan EV, Corkin S, Growdon JH. Remote memory function in Alzheimer's disease and Parkinson's disease. Brain 1988;111:185-206.

5 Sahakian BJ, Morris RG, Evenden JL, Heald A, Levy R, Philpot M, Robbins TW. A comparative study of visuospatial memory and learning in Alzheimer type dementia and Parkinson's disease. Brain 1988;111:695-718.

6 Lees AJ, Smith E. Cognitive deficits in the early stages of Parkinson's disease. Brain 1983;106:257-70.

7 Taylor AE, Saint-Cyr JA, Lang AE. Frontal lobe dysfunction in Parkinson's disease. The cortical focus of neostriatal outflow. Brain 1986;109:845-83.

8 Boller F, Passafiume D, Keefe NC, Rogers K, Morrow L, Kim Y. Visuospatial impairment in Parkinson's disease: role of perceptual and motor factors. Arch Neurol 1984;41:485-90.

9 Albert M, Feldman RG, Willis AL. The 'subcortical dementia' of progressive supranuclear palsy. $\exists$ Neurol dementia' of progressive supranuclear
Neurosurg Psychiatry 1974;37:121-30.

10 Cummings JL. Subcortical dementia: Neuropsychology, neuropsychiatry and pathophysiology. $\mathrm{Br} \mathcal{F}$ Psychiatry 1986;149:682-97.

11 Maher ER, Smith EM, Lees AJ. Cognitive deficits in Steele-Richardson-Olszewski syndrome (progressive supranuclear palsy). If Neurol Neurosurg Psychiatry 1985;48:1234-9.

12 Pillon B, Dubois B, Ploska A, Agid Y. Severity and specificity of cognitive impairment in Alzheimer's Huntington's and Parkinson's diseases and progressive supranuclear palsy. Neurology 1991;41:634-43.

13 Dubois B. Pillon B, Legault F, Agid Y, L'Hermitte F. Slowing of cognitive processing in progressive supranuclear palsy: A comparison with Parkinson's disease. Arch Neurol 1988;45:1194-9.

14 Brooks DJ, Ibanez V, Sawle GV, et al. Differing patterns of striatal 18-F Dopa uptake in Parkinson's disease, multiple system atrophy and progressive supranuclear multiple system atrophy and prog

15 Quinn N. Multiple system atrophy-the nature of the beast. I Neurol Neurosurg Psychiatry 1989;52:Suppl: beast. $\mathcal{F}$

16 Oppenheimer D. Neuropathology of progressive autonomic failure. In: Bannister R, ed. Autonomic failure, a textbook of clinical disorders of the autonomic nervous system. Oxford: Oxford University Press, 1982;267-83. 17 Berciano J. Olivopontocerebellar atrophy. A review of 
117 cases. 7 Neurol Sci 1982;53:253-72.

18 Downes JJ, Roberts AC, Sahakian BJ, Evenden IL, Morris RG, Robbins TW. Impaired extra-dimensional shift performance in medicated and unmedicated Parkinson's disease: evidence for a specific attentional dysfunction Neuropsychologia 1989;27:1329-43.

19 Owen AM, Roberts AC, Polkey CE, Sahakian BJ Robbins TW. Extradimensional versus intradimensiona set shifting performance following frontal lobe excisions, temporal lobe excisions or amygdala-hippocampectomy in man. Neuropsychologia 1991;29:993-1006.

20 Shallice T. Specific impairments in planning. In Broadbent DE, Weiskrantz L, eds. The neuropsycholog of cognitive function. London: The Royal Society, 1982, 199-209.

21 Owen AM, Downes JJ, Sahakian BJ, Polkey CE, Robbins TW. Planning and spatial working memory following frontal lobe lesions in man. Neuropsychologia 1990;28: 1021-34.

22 Morris RG, Downes J, Evenden J, Sahakian BJ, Heald A Robbins TW. Planning and spatial working memory in Parkinson's disease. $\mathcal{F}$ Neurol Neurosurg Psychiatry in Parkinson's

23 Sahakian BJ, Owen AM. Computerized assessment in neuropsychiatry using CANTAB: discussion paper. f $R$ Soc Med 1992;85:399-402.

24 Owen AM, James M, Leigh PN, et al. Fronto-striatal cognitive deficits at different stages of Parkinson's disease. Brain 1992;115:1727-51.

25 Robbins TW, James M, Lange KW, Owen AM, Quinn NP, Marsden CD. Cognitive performance in multiple system atrophy. Brain 1992;115:271-91.

26 Hoehn MM, Yahr MD. Parkinsonism: onset, progression and mortality. Neurology 1967;17:427-42.

27 Folstein MF, Folstein SE, McHugh PR. Minimental State. F Psychiat Res 1975;12:189-98.

28 Kendrick DC. Kendrick cognitive tests for the elderly. Windsor: NFER-Nelson, 1985.

29 Lees AJ. The Steele-Richardson-Olszewski syndrome (progressive supranuclear palsy). In: Marsden CD, Fahn S, eds. Movement Disorders 2. London Butterworths, 1987;272-87.

30 Neslon HE. National Adult Reading Test Manual. Windsor: Berkshire: NFER-Nelson, 1982.

31 Milner B. Interhemispheric differences in the localization of psychological processes in man. $\mathrm{Br}$ Med Bull 1971;27:272-7.
32 Roberts AC, Robbins TW, Everitt BJ. The effects of intradimensional and extradimensional shifts on visual discrimination in humans and non-human primates. $Q F$ discrimination in humans and

33 Sahakian BJ, Downes JJ, Eagger S, et al. Sparing of attentional relative to mnemonic function in a subgroup of patients with dementia of the Alzheimer type Neuropsychologia 1990;28:1 197-213

34 Lange KW, Robbins TW, Marsden CD, James M, Owen AM, Paul GM. L-Dopa withdrawal in Parkinson's disease selectively impairs cognitive performance in tests sensitive to frontal lobe dysfunction. Psychopharmacology 1992;107:394-404.

35 Winer BJ. Statistical Principles in Experimental Design, 2nd edn. New York: McGraw-Hill, 1971.

36 Kullback S. Information Theory and Statistics. New York: Dover, 1968.

37 Robbins TW. A critique of the methods available for the measurement of spontaneous locomotor activity. In: measurement of spontaneous locomotor activity. In: Psychopharmacology Vol 7. New York: Plenum Press, Psychopharmacol

38 Grafman J, Litvan I, Gomez C, Chase TN. Frontal lobe function in progressive supranuclear palsy. Arch Neurol 1990;47:553-8

39 Blin J, Baron JC, Dubois B, Pillon B, Cambon H, Cambier J, Agid Y. Positron emission tomography study in progressive supranuclear palsy: brain hypometabolic pattern and clinicometabolic correlation. Arch Neurol 1990;47:747-52.

40 D'Antona R, Baron JC, Samson Y, et al. Subcortical dementia: frontal cortex hypometabolism detected by positron tomography in patients with progressive supranuclear palsy. Brain 1985;108:785-99.

41 Goffinet A, De Volder AG, Gillian C, et al. Positron tomography demonstrates frontal lobe hypometabolism in progressive supranuclear palsy. Ann Neurol 1989;25. in prog

42 Sahgal A, Lloyd S, Wray CJ, et al. Does visuospatial memory in Alzheimer's disease depend on the severity of the disorder? Int $f$ Ger Psychiatry 1992;7:427-36.

43 Alexander GE, DeLong MR, Strick PL. Parallel organization of functionally segregated circuits linking basal ganglia and cortex. Annu Rev Neurosci 1986;9:357-81.

44 Owen AM, Beksinska M, James $M$, et al. Visuospatial memory deficits at different stages of Parkinson's disease. Neuropsychologia 1993;31:627-44. 